\title{
In memoriam Philippe Leuenberger
}

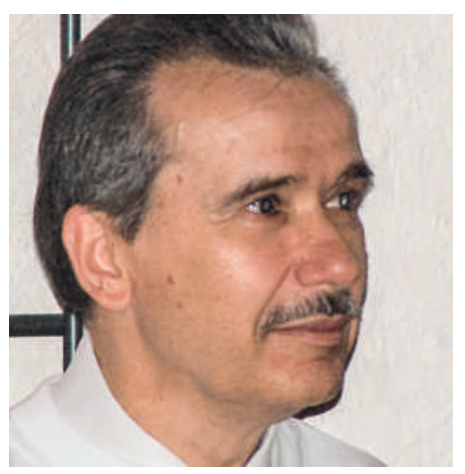

Philippe Leuenberger (1944-2015)
C'est avec beaucoup d'émotion et de tristesse que les pneumologues suisses ont appris la disparition le 28 septembre dernier du Professeur Philippe Leuenberger. Chef du Service de Pneumologie du CHUV de 1982 à 2007, Philippe Leuenberger a été une figure marquante de la pneumologie de notre pays.

Après avoir effectué sa formation médicale à l'Université de Lausanne en 1969, il accomplit sa spécialisation en maladie respiratoire auprès du Prof. Favez, puis un stage postdoctoral de deux ans à la Columbia University, New York. De retour à Lausanne, il accompagna le déménagement du service des hauteurs de Sylvana dans le nouveau bâtiment hospitalier du CHUV, en conduisant en parallèle l'évolution de cette spécialité, de l'ancienne phtysiologie vers une discipline moderne, interactive et connectée aux grands problèmes de santé publique que sont l'asthme et la BPCO. Son rayonnement l'amena à présider, entre autres, la Société Suisse de Pneumologie et d'être choisi comme organisateur du congrès de l'European Respiratory Society en 1999 à Genève.

Il fut également pionnier dans le domaine pédagogique, étant un des premiers à concevoir un enseignement assisté par CD-ROM dans le courant des années 80 . A de nombreuses reprises, les étudiants de la Faculté de Médecine l'ont élu «meilleur enseignant de l'année».

Mais sa grande œuvre restera sans conteste l'étude SAPALDIA, vaste cohorte réunissant quelque 10000 participants de toute la Suisse dont les données respiratoires ont été collectées au début des années 90 puis à nouveau en 2000. Grâce à la perspicacité et au leadership de Ph. Leuenberger, cette étude épidémiologique, unique à l'échelle de notre pays, a permis de nombreuses découvertes sur les effets respiratoires de la pollution atmosphérique et a gagné très vite une réputation internationale. Ce succès a permis de poursuivre l'étude pour une nouvelle décennie, à nouveau couron- née de nombreuses et prestigieuses publications. A l'heure actuelle, le projet SAPALDIA, toujours actif, entame sa troisième décennie.

Puis au début des années 2000 sont apparus peu à peu des symptômes révélant que le corps se refusait insidieusement d'obéir à son maître. Les progrès de la maladie obligèrent Philippe Leuenberger à quitter prématurément le service de pneumologie, étant parvenu à l'extrême limite de ses forces. Le qualificatif qui résume le mieux la personnalité de ce gentleman est celui «d'élégance». Non pas seulement une élégance

\section{Sa grande œuvre restera sans conteste l'étude} SAPALDIA.

de surface mais surtout une qualité et une chaleur dans le contact qui étaient unanimement appréciées. Alors que ces derniers temps la souffrance laissait des traces ostensibles sur son visage, son regard conservait cette fraîcheur et cet émerveillement qui l'ont toujours caractérisé.

Philippe Leuenberger savait que la science médicale est un domaine mouvant et que les certitudes d'aujourd'hui seraient très certainement remises en doute demain. Aussi a-t-il pris le parti tout au long de sa carrière de faire partager non pas uniquement un savoir ou un savoir-faire mais avant tout son savoir-être. Ses élèves ne peuvent que lui être reconnaissants d'avoir reçu un tel enseignement durable.

A ses proches qui l'ont secondé pendant sa carrière professionnelle et qui l'ont soutenu pendant la maladie nous exprimons toute notre sympathie.

Prof. John-David Aubert, Jean-William Fitting, Laurent P. Nicod, Service de Pneumologie, CHUV Lausanne 